\title{
Intervenciones cognitivo conductuales para diabéticos en México
}

\author{
Joel Omar González-Cantero, ' Roberto Oropeza Tena'
}

Actualización por temas

\section{ABSTRACT}

\section{Background}

Diabetes mellitus (DM) is highly prevalent in Mexico. It is estimated that only a quarter of such cases are metabolically controlled. Control of DM requires effective interventions, such as cognitive behavioral therapy.

\section{Objective}

To conduct a subject review of cognitive behavioral interventions used in Mexico to improve the control of DM.

\section{Method}

Cognitive behavioral interventions for the review were obtained from databases (Redalyc, Dialnet, SciELO), the TESIUNAM Catalog, specialist libraries, books, references of the analyzed studies, and consultations with experts. Studies carried out between 1990 and 2014 were included. Certain variables related to the intervention were analyzed; to evaluate the methodological characteristics, the criteria set by the CONSORT (Consolidated Standards of Reporting Trials) was used.

\section{Results}

Nineteen studies that met the inclusion criteria were included. Eleven interventions were looking for improved adherence to treatment and/or self-care, 11 were looking for improved mood, and 10 were looking for better quality of life and/or psychological well-being. Of the 19 studies, 18 reported favorable results for control of DM. Only two studies met with more than $50 \%$ of the criteria suggested by the CONSORT

\section{Discussion and conclusion}

The scope of the interventions is short because study samples averaged just 26.21 participants and follow-up periods averaged 2.15 months. Most of the studies do not or only partially meet with CONSORT criteria, putting into question the effects achieved in the interventions.

Key words: Interventions studies, cognitive behavioral therapy, diabetes mellitus.

\section{RESUMEN}

\section{Antecedentes}

La diabetes mellitus (DM) tiene una alta prevalencia en México. Se estima que sólo una cuarta parte de los casos está metabólicamente controlada. Se requiere de intervenciones eficaces que coadyuven a controlarla, como las cognitivo conductuales.

\section{Objetivo}

Realizar una revisión por temas de las intervenciones cognitivo conductuales utilizadas en México para mejorar el control de la DM.

\section{Método}

Las intervenciones cognitivo conductuales para la revisión se obtuvieron de bases de datos (Redalyc, Dialnet, SciELO), el Catálogo TESIUNAM, centros de documentación especializados, libros, referencias de los estudios analizados y consulta a expertos. Se incluyeron trabajos realizados entre 1990 y 2014. Se analizaron variables relacionadas con la intervención y para evaluar las características metodológicas se utilizaron los criterios del CONSORT (Consolidated Standards of Reporting Trials / Estándares Consolidados de Reporte de Ensayos).

\section{Resultados}

Fueron incluidos diecinueve estudios que cumplían con los criterios necesarios. 11 intervenciones buscaban mejorar la adherencia al tratamiento y/o al autocuidado; 11 mejorar el estado de ánimo y 10 buscaban aumentar la calidad de vida y/o bienestar psicológico. De los 19 estudios, 18 reportaron resultados favorables para el control de la DM. Sólo dos estudios cumplieron con más del $50 \%$ de los criterios sugeridos por el CONSORT.

\section{Discusión y conclusión}

Los alcances de las intervenciones son cortos, ya que las muestras de los estudios apenas promedian 26.21 participantes y los periodos de seguimiento promedian 2.15 meses. La mayoría de los estudios no cumple (o solo parcialmente) con los criterios del CONSORT, lo cual pone en discusión los efectos alcanzados en las intervenciones.

Palabras clave: Estudios de intervenciones, terapia cognitivo conductual, diabetes mellitus.

Facultad de Psicología. Universidad Michoacana de San Nicolás de Hidalgo.

Correspondencia: Mtro. Joel Omar González-Cantero. Facultad de Psicología, Universidad Michoacana de San Nicolás de Hidalgo, Artilleros de 1847, 438, Chapultepec Sur, 58260, Morelia, Mich. México. Tel: (+52 443) 317 - 1729. E-mail: joelomar.gc@gmail.com

Recibido primera versión: 15 de octubre de 2015. Segunda versión: 1 de diciembre de 2015. Tercera versión: 16 de febrero de 2016. Cuarta versión: 1 de marzo de 2016. Aceptado: 1 de marzo de 2016. 


\section{ANTECEDENTES}

La Diabetes Mellitus (DM) es una enfermedad crónica no transmisible (ECNT) con una alta prevalencia en México; de acuerdo con la Federación Internacional de Diabetes (FID), ${ }^{1}$ mientras en América Central y América del Sur es de 8.1\%, en México es de $11.92 \%$. Así, las muertes causadas por la enfermedad durante el 2013 en el país, en personas de entre 20 y 79 años, llegaron a las 70281 . $^{1}$

La DM genera complicaciones que afectan la calidad de vida de las personas y provoca problemas a sus familias. Además, genera grandes gastos para los sistemas sanitarios. Por ejemplo, se calcula que en México, ${ }^{2}$ durante el 2010, se invirtieron, aproximadamente diez mil millones de pesos en la atención de costos directos (consulta/diagnóstico, medicamentos, hospitalización, complicaciones), e indirectos (mortalidad prematura, discapacidad permanente, discapacidad temporal).

Sin embargo, el mayor problema no estriba en destinar estas grandes cantidades de dinero, sino en la ineficacia del sistema de salud, ya que el número de pacientes que alcanzan los parámetros adecuados de control metabólico es casi nulo. ${ }^{3}$

Según el análisis ${ }^{4}$ de los datos que arrojó la Encuesta Nacional de Salud y Nutrición (ENSANUT) 2012 respecto a la DM, se estima que el $21.7 \%$ de los encuestados reportaron seguir una vigilancia acuciosa del control metabólico por medio de la determinación de glucosa en sangre, y el $7.7 \%$ por el de la hemoglobina glucosilada (HbA1C). En el mismo estudio, se identificó que sólo el $25 \%$ de las personas con la enfermedad estaban controladas metabólicamente en ese momento, $24.7 \%$ en riesgo alto ( $\mathrm{HbA} 1 \mathrm{c}$ entre $7 \%$ y $9 \%$ ) y $49.8 \%$ en riesgo muy alto $(\mathrm{HbA} 1 \mathrm{c}>9 \%)$.

El tratamiento de la DM implica modificar comportamientos relacionados con la alimentación y la actividad física, tarea que resulta difícil, por lo que el abandono del tratamiento es constante. Además, en muchos casos está presente la comorbilidad con depresión y estrés, ${ }^{5}$ los cuales son obstáculos para seguir con el tratamiento.

Así, se han utilizado diversas estrategias para mejorar el control de la DM. En los reportes científicos al respecto, se identifica la predilección por intervenciones educativas; ${ }^{6-9}$ sin embargo, a pesar de que los resultados reportados son favorables en cuanto a la adquisición de conocimientos sobre la enfermedad, ${ }^{10}$ los cambios no persisten después de cierto tiempo y, además, no existe un abordaje completo de las habilidades requeridas para alcanzar el control metabólico. Para lograrlo, es necesario que las personas se entrenen en habilidades de autocuidado, adherencia al tratamiento e identificación de barreras, así como de afrontamiento de situaciones estresantes. ${ }^{11}$

En el ámbito psicológico, el enfoque cognitivo conductual ha mostrado eficacia para que las personas con DM desarrollen las habilidades (de autocuidado, adherencia al tratamiento e identificación de barreras y de afrontamiento de situaciones estresantes) necesarias para lograr el control metabólico. ${ }^{12}$ Además, este enfoque ha mostrado eficacia en el abordaje de los trastornos comórbidos como la depresión, incluso en adultos mayores, grupo etario con alta prevalencia de DM. ${ }^{13}$ Las intervenciones cognitivo conductuales se caracterizan por combinar técnicas basadas en los modelos conductuales como el condicionamiento clásico y el operante, y técnicas cognitivas, además de las del aprendizaje social.

No obstante, las intervenciones bajo este enfoque no siempre han tenido los mismos objetivos. Algunas se dirigen a dotar de habilidades para el afrontamiento del estrés y mejorar la calidad de vida:;4-19 en tanto que el propósito de otras apunta primordialmente al desarrollo de habilidades para lograr la adherencia al tratamiento. ${ }^{20-23}$ Pese a que resulte complejo, es necesario que las intervenciones se enfoquen en estrategias para el control metabólico sin soslayar la mejora de la calidad de vida de las personas con DM.

Por lo anterior, el objetivo del presente estudio fue realizar una revisión por temas de las intervenciones cognitivo conductuales realizadas para mejorar el control de la DM en México. Por un lado, las revisiones por temas pretenden sintetizar los resultados y las conclusiones de las publicaciones relacionadas con un tema dado y, por otro, analizar sus características metodológicas. Para ello se utilizarán los criterios para tratamientos no farmacológicos propuestos por el Consolidated Standards of Reporting Trials (CONSORT), ${ }^{24}$ con el afán de identificar áreas de oportunidad y hacer recomendaciones al respecto.

\section{MÉTODO}

\section{Búsqueda y selección de los estudios}

Para la búsqueda de los estudios se consideraron las siguientes fuentes: a) bases de datos informáticas (Redalyc, Dialnet, SciELO); b) catálogo TESIUNAM; c) google Académico; d) revistas especializadas (Psicología y Salud, SALUD MENTAL, Revista Mexicana de Psicología); d) libros sobre DM publicados entre 1990 y 2014; e) referencias de los estudios analizados; f) consulta a investigadores y expertos en el tema. La consulta fue a través de correo electrónico; se les pidieron datos de trabajos no publicados que cumplieran con los criterios de inclusión. En las bases de datos, la búsqueda se realizó utilizando los siguientes descriptores: "diabet***" y "cognitivo-conductual**" en título, palabras clave y contenido.

Una vez que se obtuvieron los documentos se seleccionaron aquellos trabajos que reunían los siguientes criterios de inclusión: 1. referirse a la adherencia al tratamiento y/o al autocuidado, al control metabólico, al estado de ánimo, a la calidad de vida y/o al bienestar psicológico en DM; 2. reali- 
zados en México con población mexicana; 3. elaborados entre 1990 y 2014; 4. ejecutados con base en técnicas cognitivo conductuales; 5. diseños preprueba-posprueba; 6. diseños de grupo (se descartan los diseños $\mathrm{N}=1$ ). Este proceso de búsqueda dio lugar a 19 estudios que cumplieron con todos estos criterios.

\section{Codificación de los estudios}

Los estudios seleccionados tienen características diferenciales relacionadas con las intervenciones, la enfermedad y las características de las personas con DM que participan en ellas. En el presente estudio se agruparon las distintas variables en: 1 . características de la intervención, 2. características del participante, 3. características contextuales, 4 . características metodológicas y 5 . características extrínsecas. ${ }^{25}$

Las características codificadas de la intervención fueron: a) la finalidad de la intervención (adherencia al tratamiento, control glucémico, estado de ánimo y calidad de vida); b) técnicas de intervención, según su fundamento teórico $^{26}$ (condicionamiento clásico, condicionamiento operante, teoría del aprendizaje social, cognitivas y de autocontrol); c) tipo de intervención (individual y grupal); d) modalidad de la intervención (presencial y telefónica); e) entrenamiento a familiares (sí o no, total de sesiones y total de horas del entrenamiento); f) comorbilidad (sí o no); g) impartición por profesionales de la salud (psicólogos, nutriólogos, médicos o enfermeros); h) duración de la intervención (en semanas, en horas por sujeto y promedio de horas por semana).

Características del participante: a) tipo de diabetes (DM1, DM2 o ambas); b) edad (promedio en años); c) sexo (\% de hombres que participan en la muestra); d) tiempo de padecimiento (en años).

Características contextuales: el lugar de tratamiento (centro de salud, clínica u hospital, o centro de diabéticos o universidades).

Características metodológicas: a) tamaño de la muestra; b) mortalidad experimental (número y porcentaje de sujetos que abandonan la intervención; c) seguimiento (en meses). Además, para la revisión del rigor metodológico, se utilizaron los criterios propuestos por el CONSORT. Publicado originalmente en 1996, es un conjunto de criterios usados para evaluar la presentación de reportes de investigación de ensayos controlados aleatorios, propuesto por un grupo de profesionales de la salud, estadísticos y editores. ${ }^{27}$ Con base en los principales criterios establecidos en el CONSORT, se creó una propuesta específica para la evaluación de los ensayos aleatorios de tratamiento no farmacológico como la psicoterapia y las intervenciones conductuales. Consta de 22 ítems contenidos en cinco secciones: 1 . Título y resumen; 2. Introducción (antecedentes); 3. Método (participantes, intervenciones, objetivos, resultados, tamaño de la muestra, aleatorización-secuencia de generación, ocultamiento de la asignación, implementación, cegamiento y métodos estadísticos); 4. Resultados (flujo de participantes, implementación de la intervención, reclutamiento, datos de línea base, números analizados, datos y estimación, análisis complementarios y eventos adversos); 5) Discusión (interpretación, generalización, evidencias generales) ${ }^{24}$

Características extrínsecas: a) fecha del estudio; b) publicado (sí o no).

\section{RESULTADOS}

\section{Características de la intervención}

Respecto a la finalidad de la intervención, la mayoría de los trabajos analizados (13 de los 19) tenían más de una finalidad. Once buscaban mejorar la adherencia al tratamiento y/o el autocuidado; 11 también buscaban la mejora del estado de ánimo, predominantemente se enfocaban en depresión, ansiedad y estrés y 10 buscaban mejorar la calidad de vida y/o bienestar psicológico.

En lo que concierne a las técnicas utilizadas en las distintas intervenciones, 17 utilizaron dos o más grupos de técnicas; sólo dos trabajos reportaron el uso exclusivo de técnicas basadas en la teoría del aprendizaje social. Las técnicas más usadas fueron las cognitivas y de autocontrol (17), 15 intervenciones utilizaron las basadas en la teoría del aprendizaje social y 14 las del condicionamiento clásico. Sólo una intervención incluyó técnicas basadas en el condicionamiento operante.

El tipo de intervención grupal fue utilizado en 13 intervenciones (68.42\%); en tanto que cinco fueron individuales (26.31\%) y solamente una reportó sesiones individuales y grupales $(5.26 \%)$.

La modalidad presencial fue predominantemente la más usada (94.73\%), sólo una fue vía telefónica (5.26\%).

Cuadro 1. Características de la intervención

\begin{tabular}{lccrrrr}
\hline Variables & $\begin{array}{c}\text { Número } \\
\text { de estudios }\end{array}$ & Mínimo & Máximo & Media & $\begin{array}{c}\text { Desviación } \\
\text { estándar }\end{array}$ \\
\hline Duración (en semanas) & 19 & 4 & 30 & 12.00 & 5.99 \\
Duración (total de horas por participante) & 17 & 6 & 60 & 22.54 & 11.98 \\
Promedio de horas por semana & 17 & 1 & 4 & 1.89 & 0.72 \\
Total de sesiones familiares & 3 & 8 & 16 & 12.00 & 4.00 \\
Total de horas de las sesiones familiares & 3 & 24 & 24 & 24.00 & 0.00 \\
\hline
\end{tabular}


Cuadro 2. Características del participante

\begin{tabular}{lrrrrr}
\hline Variables & $N$ & Mínimo & Máximo & Media & D. E. \\
\hline Edad promedio (en años) & 14 & 13.25 & 72.50 & 56.54 & 14.41 \\
Sexo (\% varones) & 18 & 5.88 & 75.00 & 30.36 & 21.15 \\
Tiempo de padecimiento & 4 & 7.00 & 17.50 & 11.90 & 5.68 \\
\hline
\end{tabular}

Referente al entrenamiento a familiares, sólo tres de las 19 intervenciones lo incluyeron. El promedio de tiempo dedicado a los familiares fue de 24 horas.

En relación con la comorbilidad, nueve intervenciones $(47.36 \%)$ incluyeron participantes con enfermedades crónicas comórbidas, predominantemente hipertensión; en tanto que cinco $(26.31 \%)$ especificaron en sus criterios de exclusión no admitir pacientes con enfermedades crónicas comórbidas. Las cinco restantes $(26.31 \%)$ no especificaron si los participantes tenían DM junto con otra ECNT.

Con respecto a los profesionales de la salud que participan en las sesiones de la intervenciones, en 11 de los 19 trabajos se menciona explícitamente la participación del psicólogo. Sólo en dos estudios se indica la participación de un nutriólogo; en dos de un médico y en ninguno se menciona explícitamente la participación de un enfermero u otro profesional de la salud.

En el cuadro 1 se muestran los demás resultados de las variables relacionadas con características de la intervención.

En relación con los resultados de las intervenciones, 17 estudios reportaron cambios favorables de acuerdo con su finalidad. Considerando que la hemoglobina glucosilada (HbA1c) es el indicador más confiable del control de la DM, ocho de los nueve estudios que lo utilizaron reportaron mejoras estadísticamente significativas.

\section{Características del participante}

En lo que a esto concierne, 16 intervenciones (84.21\%) se dirigieron específicamente a la DM2, sólo una a la DM1 $(5.26 \%)$ y dos $(10.52 \%)$ de las intervenciones no especifican que tipo de DM tenían los participantes (cuadro 2).

\section{Características contextuales}

En este grupo sólo se incluyó el lugar de tratamiento. Se identificó que 13 intervenciones $(68.42 \%)$ se realizaron en instituciones sanitarias (centro de salud, clínica u hospital), cinco de ellas $(26.31 \%)$ en centros de diabéticos o universidades y en uno de los estudios (5.26\%) no se indica el lugar donde se realizó la intervención.

\section{Características metodológicas}

En lo que concierne a las características metodológicas, destaca que las muestras tuvieron un promedio de 26.21 parti-
Cuadro 3. Características metodológicas

\begin{tabular}{lcccrc}
\hline Variables & $N$ & Mínimo & Máximo & Media & D. E. \\
\hline Muestra & 19 & 8 & 120 & 26.21 & 29.12 \\
Mortalidad experimental & 17 & 0 & 80 & 8.52 & 19.43 \\
(\% de sujetos) & 19 & 0 & 6 & 2.15 & 2.52 \\
Seguimiento (en meses) & 19 &
\end{tabular}

cipantes $(\mathrm{DE}=29.12)$ y que el seguimiento que hubo para evaluar los resultados de las intervenciones, apenas rebasa los dos meses con un promedio de 2.15 meses $(\mathrm{DE}=2.52$ (cuadro 3).

\section{Criterios de rigor metodológico con base en el CONSORT}

En el cuadro 4, se muestran los porcentajes resultantes del análisis con base en los 23 indicadores propuestos por el CONSORT para los tratamientos no farmacológicos. Se observa que en el rubro Título y Resumen, 11 de los estudios omiten señalar la forma de asignación de los participantes a las intervenciones, no describen las características del tratamiento, no señalan quiénes lo imparten y los datos del cegamiento en su caso; en tanto que ocho estudios sí mencionan los datos anteriores, al menos de manera parcial.

Respecto a los criterios de selección de los participantes, 18 de los 19 estudios los mencionan parcialmente. Se omite regularmente algún criterio para la elección de los escenarios de donde serán reclutados los participantes, pues no indican los motivos por los cuales se elige un hospital, un centro de salud, un club de diabéticos o una universidad. Además, en este punto, de acuerdo con el CONSORT, se deben señalar los criterios (grado académico, experiencia en años, etc.) para la elección de aquellos profesionales de la salud que aplicaron las intervenciones, dato que también se omite frecuentemente.

En torno a los criterios para la evaluación de los profesionales que aplican la intervención, sólo uno de los 19 estudios menciona cómo se evalúa su adherencia al protocolo de intervención. Sólo un estudio menciona haber asignado aleatoriamente a los participantes, y en su caso, a quienes aplicaron la intervención a los distintos grupos. Ningún estudio menciona quién realizó la secuencia de aleatorización y tampoco si hubo algún cegamiento. En aras de mostrar la mortalidad experimental y analizar mejor los resultados, sólo un estudio coloca un diagrama de flujo de los participantes o menciona cómo fueron asignados aleatoriamente, quiénes recibieron la intervención, cuántos la completaron, entre otros datos.

\section{Característica extrínsecas}

En este aspecto, 10 trabajos (52.63\%) fueron publicados en revistas de investigación y nueve $(47.36 \%)$ son trabajos de tesis de licenciatura, maestría y doctorado. 
Cuadro 4. Criterios del CONSORT

\begin{tabular}{|c|c|c|c|c|}
\hline Sección y tema & Ítem & $\%$ Presente & $\%$ Parcial & $\%$ Ausente \\
\hline Título y resumen & 1 & 0.00 & 42.11 & 57.89 \\
\hline \multicolumn{5}{|l|}{ Métodos } \\
\hline Participantes & 3 & 5.26 & 94.74 & 0.00 \\
\hline \multirow{4}{*}{ Intervenciones } & 4 & 47.36 & 21.05 & 31.57 \\
\hline & $4 a$ & 52.63 & 15.78 & 31.57 \\
\hline & $4 \mathrm{~b}$ & 15.78 & 15.78 & 68.42 \\
\hline & $4 c$ & 5.26 & 0.00 & 94.73 \\
\hline Objetivos & 5 & 36.84 & 57.89 & 5.26 \\
\hline Datos & 6 & 57.89 & 42.10 & 0.00 \\
\hline Tamaño de la muestra & 7 & 15.78 & 57.89 & 26.31 \\
\hline Aleatorización-secuencia de generación & 8 & 5.26 & 0.00 & 94.73 \\
\hline Ocultamiento de la asignación & 9 & 5.26 & 10.52 & 84.21 \\
\hline Implementación & 10 & 0.00 & 0.00 & 100.00 \\
\hline \multirow[t]{2}{*}{ Cegamiento (enmascaramiento) } & $11 a$ & 0.00 & 10.52 & 89.47 \\
\hline & $11 b$ & 0.00 & 0.00 & 100.00 \\
\hline Métodos estadísticos & 12 & 31.57 & 26.31 & 42.10 \\
\hline Resultados flujo de participantes & 13 & 5.26 & 0.00 & 94.73 \\
\hline Implementación de la intervención & 14 & 10.52 & 26.31 & 63.15 \\
\hline Reclutamiento & 15 & 0.00 & 0.00 & 100.00 \\
\hline Datos de línea base & 16 & 15.78 & 26.31 & 57.89 \\
\hline Números analizados & 17 & 57.89 & 26.31 & 15.78 \\
\hline Datos y estimación & 18 & 57.89 & 26.31 & 15.78 \\
\hline Análisis complementarios & 19 & 31.57 & 5.26 & 63.15 \\
\hline Eventos adversos & 20 & 0.00 & 5.26 & 94.73 \\
\hline Discusión Interpretación & 21 & 5.26 & 42.10 & 52.63 \\
\hline Generalización & 22 & 10.52 & 57.89 & 31.57 \\
\hline Evidencias generales & 23 & 47.36 & 47.36 & 5.26 \\
\hline
\end{tabular}

\section{DISCUSIÓN Y CONCLUSIÓN}

Este trabajo aporta un análisis de las intervenciones cognitivo conductuales realizadas en México para el control de la DM. Todos los estudios reportan resultados favorables, independientemente de su finalidad. No obstante, el análisis del cumplimiento de los criterios metodológicos indica que 17 de los 19 estudios no cumplen siquiera el 30\% de los criterios sugeridos por el CONSORT, por lo cual los efectos son cuestionables.

Con base en los resultados, se observa una tendencia a las intervenciones breves; sin embargo, resultaría relevante comparar el efecto de intervenciones breves y largas.

Pese a que, en primera instancia, sería deseable incluir la participación de los familiares en las intervenciones, pues podría mejorar los efectos, es necesario desarrollar trabajos que comparen intervenciones con y sin la participación ya que hay estudios que apuntan que dicha participación no influye o no favorece a la persona que padece DM en términos del control metabólico de la enfermedad. ${ }^{28,29}$

Otro factor que debe considerarse en futuras intervenciones, es la participación mayoritaria de las mujeres, pues en promedio apenas el $30.36 \%$ de los participantes fueron varones. Esto puede darnos datos sesgados de la efectivi- dad de las intervenciones, pues en una enfermedad como la DM, las actitudes favorables al tratamiento pueden explicar su mayor adherencia; entonces, no sería la intervención la que explique los resultados, sino la disposición de las personas, que aunque es necesaria, no es suficiente. Por ello, se recomienda la paridad en los sexos de las distintas muestras de otros estudios para identificar diferencias que pudieran resultar significativas y que las intervenciones posteriores se ajusten a las problemáticas, muy particulares, que enfrentan los hombres y las mujeres respecto al tratamiento de la DM.

Sería importante considerar la eficacia de las intervenciones en función del lugar donde se desarrollan. Esto resulta relevante porque, aunque son los escenarios sanitarios los más utilizados para revisión de su salud y la aplicación de las intervenciones, podría evaluarse si existen diferencias en los resultados, dependiendo de que se desarrollen en un hospital, un centro de salud o un club de diabéticos.

Por otra parte, el tamaño de las muestras es un criterio relacionado con la precisión estadística, y por ende con la confiabilidad de los datos, por lo cual es deseable que se aumente en futuros trabajos.

El CONSORT recomienda enfáticamente que el criterio "Título y Resumen" se cumpla cabalmente, lo cual implica 
que se indique el dato de la asignación de los participantes a los grupos de intervención en el título; que en el resumen se describa el tratamiento experimental, las comparaciones entre grupos experimentales, las características de los profesionales de la salud que imparten las intervenciones, los datos de los centros donde se imparten y el tipo de cegamiento del estudio (en su caso); esto porque este criterio es muy útil para actividades de investigación como la búsqueda y catalogación bibliográfica.

Los estudios analizados regularmente se componen de muestras no probabilísticas elegidas por conveniencia; por lo que se recomienda que estudios posteriores elijan aleatoriamente la muestra para controlar ese sesgo.

La mayoría de las intervenciones tienen claramente definidas sus poblaciones, lo cual es necesario para obtener los mejores resultados clínicamente. Esto se refleja en que 16 de los 19 trabajos se enfocan específicamente en la DM2, aunque no hay que soslayar que esta enfermedad tiene mucha comorbilidad con la hipertensión arterial y otras enfermedades, de modo que las intervenciones tienen que considerar también estos factores en su diseño.

Este trabajo abate parcialmente el fenómeno llamado "literatura gris", que se refiere al conjunto de documentos que se publican pero que se distribuyen a través de canales poco convencionales (catálogos de tesis). Así, gracias a los catálogos de tesis de las universidades y la consulta con expertos se obtuvieron nueve trabajos.

Derivado del análisis de los 19 trabajos, se evidencia la necesidad de generar intervenciones psicológicas para abordar la DM con mayor control metodológico. De lograrse intervenciones más eficaces y de mayor alcance en el control de la enfermedad, las personas podrían gozar de una mejor calidad de vida y el sistema de salud podría beneficiarse al reducir los costos asignados a la atención de las complicaciones por DM.

Es necesario evaluar el efecto de las intervenciones con sus componentes terapéuticos planteados, pero con mayor control metodológico, en períodos de seguimiento más largos y con muestras más grandes; además de evaluar sus efectos en otros indicadores clínicos como el colesterol, triglicéridos, no sólo la $\mathrm{HbA1c}$. Esto es fundamental porque en los últimos años hay una creciente comorbilidad entre los problemas cardiovasculares y la DM que termina provocando muchas muertes prematuras.

Debe buscarse la manera en que las intervenciones que prueben su eficacia se diseminen, se incorporen o adapten al sistema público de salud en aras de que sea mayor la población que pueda beneficiarse. Trabajos como el de Hattori ${ }^{30}$ y Robles $^{31}$ han mostrado solidez metodológica.

Una limitación del presente estudio es que, pese a que la literatura señala que el enfoque cognitivo conductual es el más utilizado y eficaz para abordar la DM, puede que haya trabajos con base en distintas perspectivas teóricas que hayan tenido una eficacia similar o mayor, por lo cual sería recomendable realizar algún metaanálisis con trabajos que tengan solidez metodológica para identificar claramente la eficacia de unos y otros.

\section{Financiamiento}

Ninguno.

\section{Conflicto de intereses}

Los autores declararon no tener ningún conflicto de intereses.

\section{Agradecimiento}

Al Consejo Nacional de Ciencia y Tecnología, quien otorga una beca de manutención al primer autor, gracias a la cual se pudo desarrollar este estudio.

\section{REFERENCIAS}

1. International Diabetes Federation. Atlas de la Diabetes de la FID 6 edición. Informe 2014. Belgica, Bruselas. Última consulta el 10 de octubre de 2015. http://www.idf.org/sites/default/files/Atlas-poster-2014 ES.pdf

2. Arredondo A, De Icaza E. Costos de la diabetes en América Latina: Evidencias del caso mexicano. Value Health 2011;14(5):85-88.

3. Figuerola D. Implicación del paciente con diabetes tipo 2 en el autocuidado de su enfermedad: un reto pendiente. Av Diabetol 2009;25:501502.

4. Flores S, Reyes H, Villalpando S, Reynoso N et al. Encuesta Nacional de Salud y Nutrición. Evidencia para la política pública en salud. Diabetes en adultos: urgente mejorar la atención y el control. Informe 2012. México, DF. Última consulta 12 de octubre del 2015. http://ensanut.insp.mx/doctos/analiticos/DiabetesMellitus.pdf

5. Colunga-Rodríguez C, García de Alba JE, González MA, Salazar-Estrada JG. Caracterización psicosocial de pacientes con diabetes tipo 2 en atención primaria. Rev Cub Salud Publica 2008;34(4):1-7.

6. Barceló A, Robles S, White F, Jaude L et al. Una intervención para mejorar el control de la diabetes en Chile. Rev Panam Salud Publica 2001;10(5):328-333.

7. Cervantes MA, García-Talavera NV, Brotons J, Núñez MA et al. Psychoeducative groups help control type 2 diabetes in a primary care setting. Nutr Hosp 2013;28(2):497-505.

8. Lange I, Campos S, Urrutia M, Bustamante C et al. Efecto de un modelo de apoyo telefónico en el auto-manejo y control metabólico de la diabetes tipo 2, en un Centro de Atención Primaria, Santiago, Chile. Rev Med Chile 2010;(138):729-737.

9. Lin C, Anderson RM, Hagerty BM, Lee B. Diabetes self-management experience: a focus group study of Taiwanese patients with type 2 diabetes. J Nurs Health Chronic Illn 2007;17(5):34-42.

10. Cabrera CE, Novoa A, Centeno NM. Conocimientos, actitudes y prácticas dietéticas en pacientes con diabetes mellitus II. Salud Pública Mex 1991;33(2):166-172.

11. Castro G, Rodríguez I, Ramos RM. Intervención psicológica en pacientes con diagnóstico de diabetes mellitus. Rev Facultad Ciencias Salud 2005;2(2):147-150.

12. Sánchez JD. Psicoterapia cognitivo-conductual en pacientes con diabetes mellitus tipo II. En: Hernández NA, Sánchez JD (eds.). Manual de psicoterapia cognitivo-conductual para trastornos de la salud. México: LibrosEnRed; 2007.

13. García-Peña C, Vázquez-Estupiñan F, Avalos-Pérez F, Robles LV et al. Clinical effectiveness of group cognitive-behavioural therapy for depressed older people in primary care: A randomised controlled trial. Salud Ment 2015;38(1):33-39. 
14. Del Castillo A, Guzmán R, García M, Martínez C. Intervención cognitivo-conductual para modificar el nivel de distrés en pacientes con diabetes tipo II. En: Galán S, Camacho E (eds.). Estrés y salud. México: El Manual Moderno; 2012.

15. Delgado LC, Hidalgo GA, Villalobos FH. Efectos de un programa cognitivo comportamental sobre los niveles de estrés y glucemia en pacientes con diabetes mellitus tipo II. Rev Universidad Salud 2011;2(14):31-42.

16. Riveros A, Cortazar-Palapa J, Alcazar F, Sánchez-Sosa JJ. Efectos de una intervención cognitivo-conductual en la calidad de vida, ansiedad, depresión y condición médica de pacientes diabéticos e hipertensos esenciales. International J Clinical Health Psychology 2005;5(3):445462.

17. Roselló JM, Jiménez-Chafey, MI. Cognitive-Behavioral Group Therapy for Depression in Adolescents with Diabetes: A Pilot Study. Rev Interam Psicología 2006;40(2):219-226.

18. Montes R, Oropeza R, Pedroza FJ, Verdugo JC et al. Manejo del estrés para el control metabólico de personas con diabetes mellitus tipo 2 . En-claves Pensamiento 2013;7(13):67-87.

19. Ybarra JL, Orozco LA, De León AE, Vargas AC. Intervención cognitivo-conductual para la mejora del autocuidado y la calidad de vida en adolescentes con diabetes tipo 1 y sus familias. Rev Latinoam Med Conductual 2012;2(2):96-102.

20. Casillas-Mendoza AD, González-Pérez OP, Montes-Delgado R. Influence of progressive relaxation in old age adults who suffer type 2 diabetes mellitus: pilot study. Int J Hisp Psycho 2011;3(2):293-301.

21. Rodríguez ML, Renteria A, García JC. Adherencia a la dieta en pacientes diabéticos: efectos de una intervención. Summa Psicológica UST 2013;10(1):91-101.

22. Pantoja-Magallón CE, Domínguez-Guedea MT, Moncada E, Reguera ME et al. Programa de entrenamiento en habilidades de autocontrol alimenticio en personas diabéticas e hipertensas. Rev Electrónica Psicología Iztacala 2011;14(1):98-115.

23. Quiroga A. Intervención telefónica para promover la adherencia terapéutica en pacientes diabéticos con síntomas de ansiedad y depresión. Enseñanza Investigación Psicología 2012;17(2):387-403.

24. Boutron I, Moher D, Altman DG, Schulz KF et al. Extending the CONSORT statement to randomized trials of non-pharmacologic treatment: Explanation and elaboration. Ann Intern Med 2008;148(4):295309.

25. Lipsey MW. Identifying potentially interesting variables and analysis opportunities. En: Cooper HM, Hedges LV (eds). The handbook of research synthesis. Nueva York: 1994.

26. Caballo VE. Manual de técnicas de terapia y modificación de conducta. Madrid: Siglo Veintiuno de España Editores; 1991.

27. Moher D, Shulz K, Altman D. The CONSORT statement: revised recommendations for improving the quality of reports of parallel-group randomized trials. JAMA 2001;285(15):1987-1991.

28. Arredondo A, Márquez E, Moreno F, Bazán M. Influencia del apoyo social en el control del paciente diabético tipo 2. Rev Especialidades Médico-Quirúrgicas 2006;11(3):43-48.

29. Ginarte Y. La adherencia terapéutica. Rev Cub Med General Integral 2001;17(5):502-505.

30. Hattori M. Entrenamiento en competencias de afrontamiento al adulto mayor con diabetes mellitus tipo 2 y su cuidador informal [tesis doctoral]. México: Facultad de Psicología; Universidad Nacional Autónoma de México; 2014.

31. Robles R. Evaluación y modificación de la calidad de vida de personas con diabetes mellitus tipo II [tesis doctoral]. México: Facultad de Psicología; Universidad Nacional Autónoma de México; 2002. 